\title{
Co-existence of Herpes simplex virus type 2 and two other oncoviruses is associated with cervical lesions in women living with HIV in South-Western Nigeria
}

\author{
Jude Ogechukwu Okoye, ${ }^{1}$ Anthony Ajuluchukwu Ngokere, ${ }^{2}$ Charles Erinle, ${ }^{3}$ Chinenye Mbamalu ${ }^{4}$
}

1. Nnamdi Azikiwe University, Medical Laboratory Science; Babcock University, Medical Laboratory Science.

2. Nnamdi Azikiwe University, Medical Laboratory Science.

3. State Hospital Abeokuta, Family Medicine.

4. Federal Teaching Hospital Abakaliki, Microbiology Unit.

\begin{abstract}
Background: The prevalence of Herpes simplex virus type 2 (HSV-2) in cervical lesions is under-reported, especially in Human immunodeficiency virus (HIV), Epstein-Barr virus (EBV) and Human Papillomavirus (HPV) infected persons.

Objectives: This study determined the prevalence of viral mono-infections, co-infections and squamous cell intraepithelial lesions (SIL) in HIV seropositive (HIV+) and HIV seronegative (HIV-) women.

Methods: This study included HIV+ and HIV- women (105 each). Cervical smears and viral antibodies were evaluated by Papanicolaou's technique and ELISA method, respectively.

Results: The prevalence of HSV-2, HPV and EBV infections, and SIL were higher in HIV+ women (75.2, 41.9, 41 and $32.4 \%)$ than in HIV- women (45.7, 26.7, 26.7 and 13.3\%) at $\mathrm{p}<0.0001, \mathrm{p}=0.029,0.041$ and 0.002 , respectively. Higher prevalence of viral mono-infection and tri-infection was observed in HIV+ women (43.8 and 24.8\%) than in HIV- women $(27.6$ and $8.6 \%$ ) at $\mathrm{p}=0.021$, and 0.003 , respectively. The prevalence of SIL was also higher in HIV+ women with viral mono-infection, bi-infection and tri-infection $(15.2,42.9$, and 53.8\%) than in HIV- women $(6.9,12.5$, and $44.4 \%)$ at $\mathrm{p}=0.468$, 0.041 , and 0.711 , respectively.
\end{abstract}

Conclusion: This study suggests that the high prevalence of SIL in HIV+ women could be associated with viral co-infections.

Keywords: Epstein-Barr virus; human immunodeficiency virus; human papilloma virus; herpes simplex virus type 2; cervical lesion.

DOI: https://dx.doi.org/10.4314/ahs.v20i3.4

Cite as: Okoye JO, Ngokere AA, Erinle C, Mbamalu C. Co-existence of Herpes simplex virus type 2 and two other oncoviruses is associated with cervical lesions in women living with HIV in South-Western Nigeria. Afri Health Sci. 2020;20(3): 1015-1023. https://dx.doi. org/10.4314/abs.v20i3.4

\section{Introduction}

About half a million new cases of cervical cancer (Ca) are reported each year while approximately $49 \%$ of these cases result in death worldwide ${ }^{1}$. The reason for the higher prevalence of the disease in developing countries than developed countries is yet to be fully explained. There are varied risk factors associated with $\mathrm{Ca}$ but Human Papillomavirus infection remains the major risk factor. However, not all infected women develop the $\mathrm{Ca}^{2}$. In West Africa, the prevalence of HPV DNA

Corresponding author:
Jude Ogechukwu Okoye,
Nnamdi Azikiwe University, Medical
Laboratory Science;
Emails: jog.okoye@unizik.edu.ng;
judeogeokoye@gmail.com.
ORCID ID: https://orcid.org/0000-0002-7194-5592

in atypical squamous cells of unknown significance (ASCUS), low-grade squamous intraepithelial lesion (LSIL), high-grade squamous cell intraepithelial lesion (HSIL) and invasive $\mathrm{Ca}$ (ICa) is about 26, 67, 85 and $89 \%$, respectively ${ }^{3}$. However, the risk factors associated with the remaining $74,33,15$, and $11 \%$ of ASCUS, LSIL, HSIL and ICa, respectively are insufficiently accounted for. Immunosuppression, especially due HIV infection, is associated with high prevalence of cervical lesions ${ }^{4,5}$. Studies have shown that HPV clearance is lower in HIV+ women than in HIV- women ${ }^{6,7}$, such individuals are also at risk of acquiring other oncogenic viruses such as EBV and HSV-2 ${ }^{8}$. This may explain why $25 \%$ of HIV+ women with LSIL and $12-30 \%$ of HIV+ women with HSIL still develop HSIL and ICa, respectively despite receiving antiviral therapy ${ }^{9-12}$. The reason for the progression in disease state is yet to be fully explained. Smith et al. stated that HSV-2 sero-

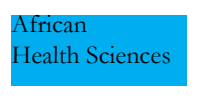

(C) 2020 Okoye JO et al. Licensee African Health Sciences. This is an Open Access article distributed under the terms of the Creative commons Attribution License (https://creativecommons.org/licenses/BY/4.0), which permits unrestricted use, distribution, and reproduction in any medium, provided the original work is properly cited. 
positive women with normal cytology have significant higher risk for the disease than HPV DNA positive women ${ }^{13}$. Some HPV $+\mathrm{Ca}$ have been found to be positive for HSV-2 antibodies ${ }^{14}$. Interestingly, Bashyai et al. observed increasing antibody titre and prevalence of HSV-2 from LSIL (11\%), HSIL (33\%) to Ca $(40 \%)^{15}$. It is believed that HSV-2 associated chronic cervicitis may facilitate EBV entry ${ }^{16}$. The pooled prevalence of EBVDNA is 3-29, 21-49, and 44-70\% in LSIL, HSIL, and $\mathrm{Ca}$, respectively ${ }^{17-21}$. This also implicates $\mathrm{EBV}$ in cervical carcinogenesis. This study determined the prevalence of viral mono-infection through tri-infection as correlates of higher SIL in HIV+ women in a developing country.

\section{Methods}

\section{Sample collection, handling and assays}

This comparative cross-sectional study was carried out between the months of April (2017) and June (2018) at Abeokuta metropolis, Ogun State, South-Western Nigeria. Considering that Ogun State prevalence survey for HIV is estimated at $1.4 \%{ }^{22}$, a total of $105 \mathrm{HIV}+$ participants were consecutively selected from the HIV Testing and Counseling Clinic at State Hospital Ijaiye, Abeokuta. This study also included 105 HIV- women; health workers and those visiting the Family Planning Clinic in the same hospital. HIV status was confirmed by testing for HIV-1 and HIV-2 antibodies in peripheral blood (using commercial kits from Qingdao Hightop Biotech Co. Ltd, China) by the ELISA method (Cutoff value $=1.854)$. According to manufacturer's instruction, we also assessed clear sera for IgG and IgM antibodies against EBV (using commercial kits from Calbiotech Inc, El Cajon, USA), HPV (using commercial kits from Qingdao Hightop Biotech Co. Ltd, China) and HSV-2 (using commercial kits from Qingdao Hightop Biotech Co. Ltd, China, Calbiotech Inc, El Cajon, USA). External genitalia were dilated using speculum and the cervix was scraped using cytobrush. The cytobrush was used in making smears and the smears were stained by Papanicolaou's and Field's techniques. The stained cervical smears were classified based on the Bethesda system: 1. Negative for intraepithelial lesion or malignancy (NILM; Normal and Cervicitis), 2. ASCUS, 3. LSIL, and 4. HSIL. Participants with abnormal Pap smear result were counseled and referred to gynecologist.

Interviewer based questionnaire was used to collect socio-economic and clinical demographics: age, marital status, family type, tribe, educational level, residency, religion, occupation, economic status (Low $<18,000$ minimum wage, middle $=18,000$ to 53000 and High $\geq$ $54,000)$ smoking status and alcohol consumption, age at sex debut, parity, sexual behaviour, medical history, number of sex partners, oral sex, type of contraceptives used, vaginal bleeding after sexual intercourse, genital ulcer, itching and burning sensation around the vulva, vaginal discharge, pelvic pain, duration of antiretroviral therapy and uptake of cervical screening.

\section{Data analysis}

The sociodemographic data obtained from the questionnaire and the test results were coded as 0 (reference) and 1 (depending on the number of sub-variable) in excel, exported into SPSS (version 23) and analyzed in descending sorting order of categorical targets. Binary logistic regression analysis was used to assess the relationship between some sub-variables. Chi-square/Fisher exact test was used to compare viral infections and Pap smear result (SIL) between HIV+ and HIV- participants in relation to some demographic characteristics. Pearson's correlation was used to assess the relationship between viral infection and cervical lesions. Significant levels were set at $\mathrm{p} \leq 0.05$.

\section{Ethical approvals}

Ethical clearances were obtained from State Hospital Abeokuta Research Ethics Services (SHA/RES/ VOL.2/147) and Babcock University Health Research Ethics Committee (BUHREC 353/16) and written informed consents were obtained from participants. All protocols were carried out in line with the guidelines of the ethics committees.

\section{Result}

This study determined the prevalence of viral mono-infections and co-infections, and cervical lesions in sexually active HIV + (mean age $=41.55 \pm 11.71$ years) and HIV- (mean age $=39.45 \pm 11.16$ years) participants $(\mathrm{p}=$ 0.08). It included HIV+ participants who were receiving highly active anti-retroviral therapy at HIV Testing and Counseling Clinic, State Hospital Ijaiye, Abeokuta. The HIV- participants were apparently healthy women with no history of cervical lesions. The prevalence of pre-cancerous lesions (ASCUS, LSIL and HSIL; figure 1) was significantly higher in HIV+ women (51.3\%) than in HIV- women $(24.8 \%)(p=0.0001)$. Overall, the prevalence of viral co-infections was higher in HIV+ women $(88.6 \%)$ than in HIV- women $(33.4 \%)$ at $\mathrm{p}<$ 0.0001. The prevalence of HSV-2, HPV and EBV infections, and SIL were higher in HIV+ women (75.2, 
41.9, 41 and 32.4\%) than in HIV- women (45.7, 26.7, 26.7 and $13.3 \%$ at $\mathrm{p}<0.0001, \mathrm{p}=0.029, \mathrm{p}=0.041$ and $\mathrm{p}=0.002)$. Result showed that there was a significant correlation between viral infection and cervical lesions both in HIV+ women $(r=0.363, p=0.000)$ and HIVwomen $(p=0.000)$. However, significant positive relationship between extent of viral infection (mono-, biand tri-infection) and cervical lesions was only seen in HIV+ women $(p=0.040)$ while insignificant positive relationship was seen in HIV- women $(p=0.326)$. Statistics revealed that the prevalence of viral mono-infection was higher in HIV+ women (43.8\%) than in HIVwomen $(27.6 \% ; \mathrm{p}=0.021)$. However, the prevalence of
SIL in viral mono-infection was insignificantly higher in HIV+ women $(15.2 \%)$ than in HIV- women $(6.9 \%$; $\mathrm{p}=$ 0.468). No significant difference was observed in the prevalence of viral bi-infection between HIV + (21\%) and HIV- women $(22.9 \% ; \mathrm{p}=0.737)$. Interestingly the prevalnce of SIL in viral bi-infection was significantly higher in HIV+ women (42.9\%) than in HIV- women $(12.5 \% ; \mathrm{p}=0.041)$. Although, the prevalence of viral tri-infection was higher in HIV+ women $(24.8 \%)$ than in HIV- women $(8.6 \% ; p=0.003)$, no significant difference was observed when the prevalence of SIL was compare between HIV+ $(53.8 \%)$ and HIV-women (44.4\%) with viral tri-infections $(\mathrm{p}=0711)$.
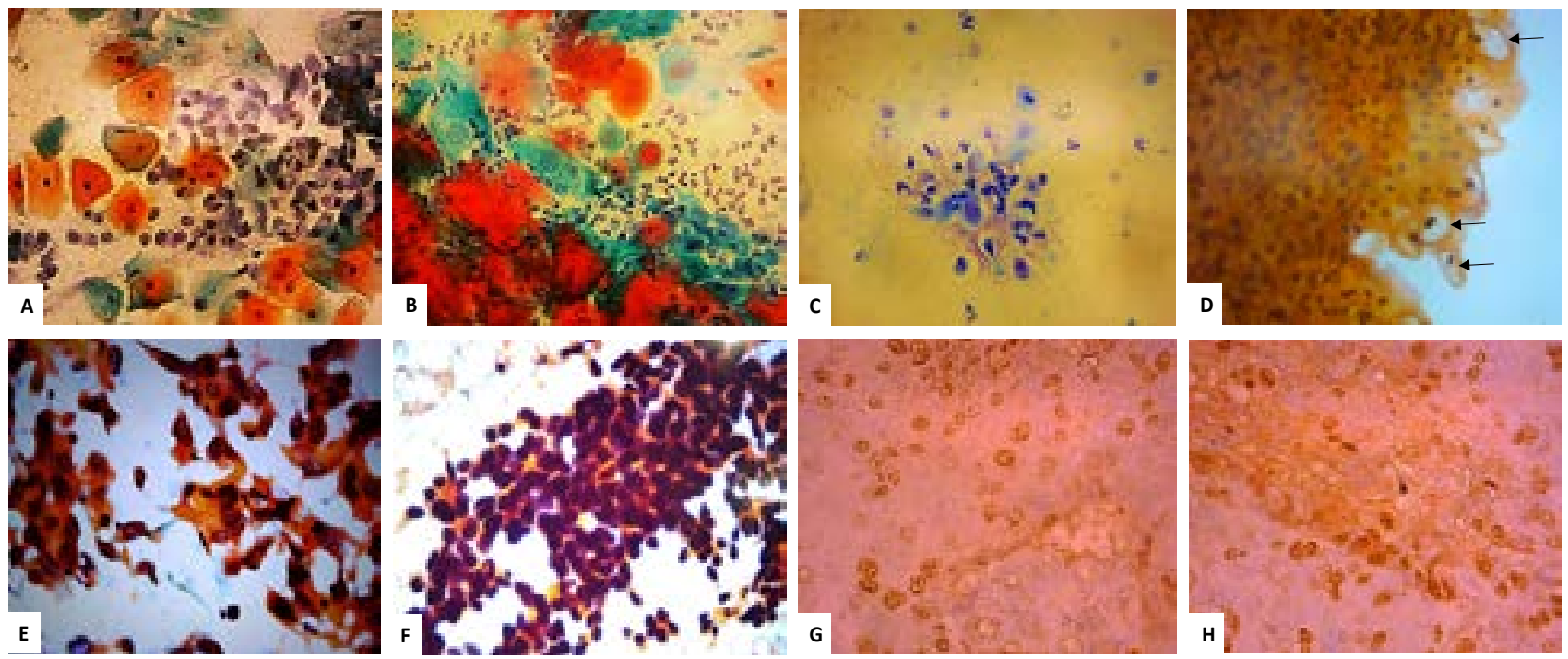

Figure 1: Photomicrographs of stained normal and abnormal cervical smears and abnormal tissue sections

Figure 1: Smear A (NILM; X100), B (ASCUS with inflammatory background; X100), D (LSIL with HPV koilocytes, marked by arrows; X400), E and F (HSIL; X400) were stained by Papanicolaou's technique while C (LSIL; X400) was stained by Field's staining technique. Section G (HSIL associated with HIV/EBV/HPV/HSV-2; X100) stain moderately positive for ki67 compared with the high staining of section H (HSIL associated with EBV/HPV/HSV-2; X100).

Influence of demographics on prevalence of SIL and viral infections

Descriptive statistics showed that the prevalence of SIL relatively increased with age both in HIV+ and HIVparticipants with the peak prevalence in the age group of 50-59 years. The prevalence of EBV infection increased with age in HIV+ participants while the prevalence of EBV infection peaked in the age group of 3039 years in HIV- participants and decreased afterwards. A significant difference in EBV infection was observed between HIV+ and HIV-participants in the age range of 50-59 years. The prevalence HPV infection relatively decreased with age in HIV+ and HIV- participants. A significant difference in HPV infection was also observed between HIV+ and HIV- participants in the age group of 30-39 years. A significant correlation was also observed between age and acute HSV-2 infection $(\mathrm{r}=0.12, \mathrm{p}=0.03)$ in both groups. The prevalence of HSV-2 infection relatively decreased with age in HIV+ participants while the prevalence of the virus changes across age groups in HIV- women but peaked in the age group of 40-49 years. Significant differences in HSV-2 infection were also observed between HIV+ and HIV-participants in the age group of 20-39 years. Among the married women and women in polygamous marriages, the prevalence of SIL, HPV and HSV-2 infections were higher in HIV+ participants than their HIV- counterparts. Bivariate analysis revealed that the risk of EBV and HPV infection among HIV+ women in polygamous marriages were 3.26 and 4.67 (95\% Cl: $0.28-38.48$ and $0.39-55.48)$ at $\mathrm{p}=0.35$ and 0.22 , respectively when compared with women who have never been married. The odd ratio for both infections were lower among HIV+ women in monogamous marriage 
0.79 and 2.00 (95\% Cl: $0.35-1.79$ and $0.89-4.51)$ at $\mathrm{p}=$ 0.58 and 0.95 , respectively when compared with women who have never been married. Among women of Yoruba tribe, HIV+ participants had a significantly higher prevalence of HPV and HSV-2 infections than their HIV- counterparts. The number of participants with post basic (secondary school) education was lower among HIV+ participants than their HIV- counterparts $(p=0.003)$. The HIV+ participants with only basic education and those living in urban areas had a significantly higher prevalence of SIL, HPV and HSV-2 infections than their HIV- counterparts. The HIV+ women who were Christians had a significantly higher prevalence of SIL, EBV and HSV-2 infections than HIV-women. Significantly higher prevalence of HPV infection was observed in HIV+ Muslims than HIV- Muslims. The prevalence of participants with low income was higher in HIV+ participants compared with HIV-participants $(p<0.0001)$. The HIV+ participants who with low income had significantly higher prevalence of SIL, EBV and HSV-2 infections than HIV- participants. The prevalence of SIL and HSV-2 infection were higher in HIV+ multiparous women than their HIV- counterparts. In $\mathrm{HIV}+$ women, although the prevalence of EBV and HPV were higher among those who had first sexual intercourse at $\leq 18$ years, these participants surprisingly had lower prevalence of SIL than those who had their sex debut at $\geq 18$ years. The HIV+ women with sex debut at $\leq 21$ years had higher prevalencef HSV-2 than their HIV- counterparts. The HIV+ debutants at $\leq 18$ years and 19-21 years had higher prevalence of EBV and HPV infections, respectively when compared with HIV- debutants. The odd ratio of HPV infection in $\mathrm{HIV}+$ debutants at 19-21 years is 44.97 (95\% Cl: 1.80-
1121) at $p=0.02$. Participants living with HIV who use only hormonal contraceptives had a significantly higher HPV infection when compared with their HIV- counterparts while $\mathrm{HIV}+$ participants who intermittently use condom had significantly higher prevalence of EBV and HSV-2 infections than their HIV- participants. The HIV + participants who had multiple sexual partners had significantly higher prevalence of SIL and HSV2 infection than their HIV- participants. Significantly higher prevalence of HSV-2 infection was observed in $\mathrm{HIV}+$ participants with vulval itching, vaginal discharge and pelvic pain than HIV- participants. The prevalence of EBV and HPV infections in HIV+ women who had HSIL were significantly higher than that of HIVwomen. The prevalence of HPV and HSV-2 is higher in HIV+ women who had cervicitis and ASCUS, respectively than in HIV- women (table 1). Among HIV+ participants, significant associations were observed between EBV infection and genital ulcers in HIV+ women $(p=0.02)$. Among HIV+ women, multivariate analysis (MANOVA) revealed significant associations between EBV infection and cervical lesions $(p=0.001)$, HSV-2 and cervical lesions $(p=0.03)$, HPV infection and cervical lesions $(p=0.001), H S V-2$ and EBV infection $(p=0.001)$, HPV and EBV infection $(p=0.001)$, and $\mathrm{EBV} / \mathrm{HPV} / \mathrm{HSV}-2$ and cervical lesions $(\mathrm{P}=0.001)$. Among HIV- participants, MANOVA revealed a significant association between EBV and HSV-2 infections $(p=0.001)$. It also revealed significant associations between EBV and Oral sex $(\mathrm{p}=0.05), \mathrm{EBV}$ infection and pelvic pain $(\mathrm{p}=0.02), \mathrm{EBV} / \mathrm{HPV} / \mathrm{HSV}-2$ tri-infection and history of multiple sex partner $(p=0.02), E B V /$ $\mathrm{HPV} / \mathrm{HSV}-2$ tri-infection and vulval itching $(\mathrm{p}=0.02)$, and cervical lesions and vaginal bleeding $(\mathrm{p}=0.03)$. 
Table 1a: Socioeconomic and clinical characteristics of HIV seropositive and seronegative participants

\begin{tabular}{|c|c|c|c|c|c|c|c|c|c|c|c|c|c|c|c|}
\hline \multirow[t]{2}{*}{ Variables } & \multirow[t]{2}{*}{ Sub-variables } & HIV+ Ppts & HIV- Ppts & \multicolumn{2}{|c|}{ Ppts with SIL } & \multirow{2}{*}{$\begin{array}{c}\mathrm{p}- \\
\text { value }\end{array}$} & \multicolumn{2}{|c|}{ EBV Infection } & \multirow{2}{*}{$\begin{array}{c}\mathrm{p}- \\
\text { value }\end{array}$} & \multicolumn{2}{|c|}{ HPV Infection } & \multirow{2}{*}{$\begin{array}{c}\mathrm{p}- \\
\text { value }\end{array}$} & \multicolumn{2}{|c|}{ HSV-2 Infection } & \multirow{2}{*}{$\begin{array}{c}\mathrm{p}- \\
\text { value }\end{array}$} \\
\hline & & $\mathrm{N}=105$ & $\mathrm{~N}=105$ & $\begin{array}{l}\text { HIV+ } \\
n=34\end{array}$ & $\begin{array}{l}\text { HIV- } \\
n=14\end{array}$ & & $\begin{array}{l}\mathrm{HIV+}+ \\
\mathrm{n}=43\end{array}$ & $\begin{array}{l}\text { HIV- } \\
n=28\end{array}$ & & $\begin{array}{l}\text { HIV+ } \\
n=44\end{array}$ & $\begin{array}{c}\text { HIV- } \\
n=28\end{array}$ & & $\begin{array}{l}\text { HIV+ } \\
n=79\end{array}$ & $\begin{array}{l}\text { HIV- } \\
\mathrm{n}=48\end{array}$ & \\
\hline \multirow[t]{5}{*}{ Age } & $20-29$ & 39 (37.1) & $38(36.2)$ & 9 (23.1) & $5(13.2)$ & 0.377 & $15(38.5)$ & $10(26.3)$ & 0.332 & $17(43.6)$ & $13(34.2)$ & 0.485 & $32(82.1)$ & $20(52.6)$ & .008 \\
\hline & $30-39$ & $31(29.5)$ & $42(40.0)$ & $9(29.0)$ & $5(11.9)$ & 0.076 & $12(38.7)$ & $14(33.3)$ & .805 & $14(45.2)$ & $8(19.0)$ & 0.021 & $23(74.2)$ & $17(40.5)$ & .009 \\
\hline & $40-49$ & $25(23.8)$ & $13(12.4)$ & $10(40.0)$ & $2(15.4)$ & 0.158 & $11(44.0)$ & $3(23.1)$ & 0.294 & $10(40.0)$ & $6(24.0)$ & 0.742 & $17(68.0)$ & 8 (61.5) & 0.730 \\
\hline & $50-59$ & $9 \quad(8.6)$ & $11(10.5)$ & $6(66.7)$ & $2(18.2)$ & 0.065 & $5(55.6)$ & 1 (9.1) & 0.050 & $3(33.3)$ & 1 (11.1) & 0.285 & $6(66.7)$ & $3(27.3)$ & 0.175 \\
\hline & $60-69$ & 1 (1.0) & $1(1.0)$ & $0(0.0)$ & $0(0.0)$ & 1.000 & $0(0.0)$ & $\begin{array}{ll}0 & (0.0)\end{array}$ & 1.000 & $0(0.0)$ & $0(0.0)$ & 1.000 & $1(100)$ & $\begin{array}{ll}0 & (0.0)\end{array}$ & 1.000 \\
\hline \multirow[t]{3}{*}{ Marital Status } & Never Married & $3(2.9)$ & 44 (41.9) & $0(0.0)$ & $7(70.5)$ & 1.000 & $2(66.7)$ & $11(25.0)$ & 0.181 & $2(66.7)$ & $18(40.9)$ & 0.567 & $3(100)$ & $22(50.0)$ & 0.237 \\
\hline & Married & $72(68.6)$ & $46(43.8)$ & $24(86.1)$ & $6(52.2)$ & 0.017 & $28(38.9)$ & $13(28.3)$ & 0.836 & $30(41.7)$ & $6(13.0)$ & 0.001 & $50(69.4)$ & $19(41.3)$ & 0.004 \\
\hline & $\mathrm{D} / \mathrm{S} / \mathrm{W}$ & $30(28.6)$ & $15(14.3)$ & $10(93.3)$ & $1(46.7)$ & 0.070 & $13(43.3)$ & $4(26.7)$ & 0.341 & $12(40.0)$ & $4(26.7)$ & 0.514 & $26(86.7)$ & $7 \quad(46.7)$ & 0.010 \\
\hline \multirow[t]{3}{*}{ Family Type } & Never Mc & $3 \quad(2.9)$ & $43(41.1)$ & $0(0.0)$ & $7(16.3)$ & 1.000 & $2(66.7)$ & $11(25.6)$ & 0.188 & $2(66.7)$ & $18(41.9)$ & 0.572 & $3(100)$ & $22(51.2)$ & 0.239 \\
\hline & Poly & $52(49.5)$ & $28(26.7)$ & $19(36.5)$ & $3(10.7)$ & 0.018 & $21(40.4)$ & $10(35.7)$ & 0.811 & 25 (48.1) & $3(10.7)$ & 0.001 & $38(73.1)$ & $12(42.9)$ & 0.015 \\
\hline & Mono & $50(47.6)$ & 34 (32.4) & $15(30.0)$ & $4(11.8)$ & 0.065 & $20(40.0)$ & 7 (20.6) & 0.095 & $17(34.0)$ & $7(20.6)$ & 0.223 & $38(76.0)$ & 14 (63.6) & 0.003 \\
\hline \multirow[t]{4}{*}{ Tribe } & Igbo & $3(2.9)$ & $22(21.0)$ & $2(66.7)$ & $3(13.6)$ & 0.091 & $2(66.7)$ & $6(27.3)$ & 0.231 & $2(66.7)$ & 7 (31.8) & 0.530 & $2(66.7)$ & $13(59.1)$ & 1.000 \\
\hline & Yoruba & $98(93.3)$ & $54(51.4)$ & 31 (31.6) & 10(18.5) & 0.089 & $40(40.8)$ & $18(33.3)$ & 0.388 & 41 (41.8) & $11(20.4)$ & 0.008 & $77(78.6)$ & $25(46.3)$ & 0.000 \\
\hline & Hausa & $2(1.9)$ & $5 \quad(4.8)$ & $0(0.0)$ & $0(0.0)$ & 1.000 & $1(50.0)$ & $0(0.0)$ & 1.000 & $1(50.0)$ & $1(20.0)$ & 1.000 & $0(0.0)$ & $1(20.0)$ & 1.000 \\
\hline & Others & $2(1.9)$ & $24(22.9)$ & $1(50.0)$ & & 0.151 & $1(50.0)$ & $6(25.0)$ & 0.474 & $0(0.0)$ & $11(45.8)$ & 0.492 & $0(0.0)$ & .5) & 1529 \\
\hline \multirow[t]{4}{*}{ Education } & N.F.E. & $11(10.5)$ & $6 \quad(5.7)$ & $3(27.3)$ & 5.7) & 1.000 & $6(54.5)$ & $1(16.7)$ & 0.304 & $5(45.5)$ & $3(50.0)$ & 1.000 & $11(100)$ & 6.7) & 0.110 \\
\hline & Primary & $54(51.4)$ & $27(25.7)$ & $20(37.0)$ & $4(14.8)$ & 0.043 & $19(35.2)$ & $8(29.6)$ & 0.803 & $20(37.0)$ & $10(37.0)$ & 1.000 & $38(70.4)$ & $15(55.6)$ & 0.220 \\
\hline & Secondary & $31(29.5)$ & $46(43.8)$ & $9(29.0)$ & $3(6.5)$ & 0.012 & $12(38.7)$ & $10(21.7)$ & 0.128 & $16(51.6)$ & $10(21.7)$ & 0.013 & $23(74.2)$ & 21( & 0.019 \\
\hline & Post-Sec & $9 \quad(8.6)$ & $26(24.8)$ & $2(22.2)$ & $6(23.1)$ & 1.000 & $6(66.7)$ & $9(34.6)$ & 0.129 & $3(33.3)$ & 9 (34.6) & 1.000 & 7 (77.8) & $12(46.2)$ & 0.135 \\
\hline \multirow[t]{2}{*}{ Residence } & Urban & $100(95.2)$ & 64 (61.0) & $32(32.0)$ & & 0.010 & 41 (64.1) & $19(29.7)$ & 0.184 & $40(40.0)$ & $15(23.4)$ & .041 & 76 (76.0) & 32 & 0.001 \\
\hline & Rural & $5 \quad(4.8)$ & $41(39.0)$ & $2(40.0)$ & $5(100)$ & 0.160 & $2(40.0)$ & $9(22.0)$ & 0.580 & $4(80.0)$ & $13(31.7)$ & 0.055 & $3(60.0)$ & $16(39.0)$ & 0.635 \\
\hline \multirow[t]{2}{*}{ Religion } & Christian & 65 (61.9) & $93(88.6)$ & $19(29.2)$ & $12(12.9)$ & 0.014 & $29(44.6)$ & $22(23.7)$ & 0.009 & $25(38.5)$ & $25(26.9)$ & 0.164 & $48(27.7)$ & $40(43.0)$ & 0.000 \\
\hline & Muslim & $40(38.1)$ & $12(11.4)$ & $15(37.5)$ & $2(16.7)$ & 0.484 & $14(35.0)$ & $6(50.0)$ & 0.500 & $19(47.5)$ & $3(25.0)$ & 0.012 & $31(37.5)$ & $8 \quad(66.7)$ & 0.466 \\
\hline \multirow[t]{3}{*}{ Income Status } & Low & 77 (73.3) & $34(32.4)$ & $25(32.5)$ & $4(11.8)$ & 0.033 & $35(45.5)$ & 7 (20.6) & 0.019 & $34(44.2)$ & $11(32.4)$ & 0.297 & $59(76.6)$ & $16(47.1)$ & 0.012 \\
\hline & Middle & $22(21.0)$ & $51(48.6)$ & $6(27.3)$ & $5(9.8)$ & 0.077 & 7 (31.8) & $15(29.4)$ & 1.000 & 7 (31.8) & $15(29.4)$ & 1.000 & $16(72.7)$ & $22(43.1)$ & 0.024 \\
\hline & High & $6 \quad(5.7)$ & $20(19.0)$ & $3(50.0)$ & $5(25.0)$ & 0.330 & 1 (16.7) & $6(30.0)$ & 0.647 & $3(50.0)$ & $2(10.0)$ & 0.062 & $4(66.7)$ & $10(50.0)$ & 0.652 \\
\hline Parity & Null & $46(43.8)$ & 39 (37.1) & $16(34.8)$ & $6(15.4)$ & 0.050 & $20(43.5)$ & 7 (17.9) & 0.019 & $18(39.1)$ & $12(30.8)$ & 0.498 & $33(71.7)$ & $17(43.6)$ & 0.015 \\
\hline & $1-2$ & $\begin{array}{ll}6 & (5.7)\end{array}$ & $8 \quad(7.6)$ & $2(33.3)$ & $2(25.0)$ & 1.000 & 1 (16.7) & $4(50.0)$ & 0.301 & $4(66.7)$ & $1(12.5)$ & 0.091 & $3(50.0)$ & $6(75.0)$ & 0.580 \\
\hline & Multiparous & $53(50.5)$ & $58(55.2)$ & $16(30.2)$ & $6(10.3)$ & 0.016 & $22(41.5)$ & $17(29.3)$ & 0.233 & $22(41.5)$ & $15(25.9)$ & 0.107 & $43(81.1)$ & $25(43.1)$ & 0.000 \\
\hline Age at sex debut & $\leq 18$ years & $26(24.8)$ & $40(38.1)$ & $6(23.1)$ & $4(10.0)$ & 0.175 & $14(53.8)$ & $8(20.0)$ & 0.007 & $13(50.0)$ & $11(27.5)$ & 0.074 & $19(73.1)$ & $17(42.5)$ & 0.023 \\
\hline & $19-21$ years & $48(45.7)$ & $43(41.0)$ & $14(29.2)$ & $6(14.0)$ & 0.127 & $17(35.4)$ & $11(25.6)$ & 0.367 & $17(35.4)$ & 5 (11.6) & 0.013 & $36(75.0)$ & $19(44.2)$ & 0.005 \\
\hline & $\geq 22$ years & $31(29.5)$ & $22(21.0)$ & $14(45.2)$ & $4(18.2)$ & 0.076 & $12(38.7)$ & 9 (40.9) & 1.000 & $14(45.2)$ & 7 (31.8) & 0.393 & $24(77.4)$ & $12(54.5)$ & 0.134 \\
\hline Contraceptive & Hormonal & $51(48.6)$ & 40 (38.1) & $15(29.4)$ & $6(15.0)$ & 0.135 & $21(41.2)$ & $13(32.5)$ & 0.513 & $21(41.2)$ & $5(12.5)$ & 0.005 & $41(80.0)$ & $18(45.0)$ & 1.000 \\
\hline & Condom & $54(51.4)$ & 65 (61.9) & $18(33.3)$ & $8(12.3)$ & 0.001 & $22(40.7)$ & $15(23.1)$ & 0.048 & $23(42.6)$ & $23(35.4)$ & 0.454 & $38(70.4)$ & $30(46.2)$ & 0.010 \\
\hline Oral Sex & No & 104(99.0) & $89(84.8)$ & 34 (32.7) & 11(12.4) & 0.001 & $42(40.4)$ & 25 (28.1) & 0.095 & $44(42.3)$ & $22(24.7)$ & 0.015 & $78(75.0)$ & $39(43.8)$ & 0.000 \\
\hline & Yes & 1 (1.0) & $16(15.2)$ & $0 \quad(0.0)$ & $3(18.8)$ & 1.000 & $1(100)$ & $3(18.8)$ & 0.235 & $0(0.0)$ & $6(37.5)$ & 1.000 & $1(100)$ & $9 \quad(56.3)$ & 1.000 \\
\hline Smoker & No & $105(100)$ & $90(85.7)$ & $34(32.7)$ & 12(13.3) & 0.002 & $43(41.0)$ & $25(27.8)$ & 0.070 & 44 (41.9) & $21(23.3)$ & 0.006 & $79(75.2)$ & $39(43.3)$ & 0.000 \\
\hline & Yes & $\begin{array}{ll}0 & (0.0)\end{array}$ & $15(14.3)$ & $0(0.0)$ & $2(13.3)$ & 1.000 & $\begin{array}{ll}0 & (0.0)\end{array}$ & $3(20.0)$ & 1.000 & $0(0.0)$ & 7 (46.7) & 1.000 & $0(0.0)$ & $9(60.0)$ & 1.000 \\
\hline Alcohol intake & No & 101(96.2) & $71(67.6)$ & $33(32.7)$ & $11(15.5)$ & 0.013 & $43(42.6)$ & $21(29.6)$ & 0.151 & $41(40.6)$ & $20(28.2)$ & 0.107 & $76(75.2)$ & 34 (47.9) & 0.000 \\
\hline & Yes & $4 \quad(3.8)$ & $34(32.4)$ & $1(25.0)$ & $3(8.8)$ & 0.372 & $2(50.0)$ & 7 (20.6) & 0.233 & $3(75.0)$ & $8(23.5)$ & 0.065 & $3(75.0)$ & $14(41.2)$ & 0.307 \\
\hline
\end{tabular}


Table 1b: Socioeconomic and clinical characteristics of HIV seropositive and seronegative participants

\begin{tabular}{|c|c|c|c|c|c|c|c|c|c|c|c|c|c|c|c|}
\hline \multirow[t]{2}{*}{ Variables } & \multirow[t]{2}{*}{ Sub-variables } & \multirow{2}{*}{$\begin{array}{l}\text { HIV+ Ppts } \\
\mathrm{N}=105\end{array}$} & \multirow{2}{*}{$\begin{array}{l}\text { HIV- Ppts } \\
\mathrm{N}=105\end{array}$} & \multicolumn{2}{|c|}{ Ppts with SIL } & \multirow{2}{*}{$\begin{array}{c}\mathrm{p}- \\
\text { value }\end{array}$} & \multicolumn{2}{|c|}{ EBV Infection } & \multirow{2}{*}{$\begin{array}{c}p- \\
\text { value }\end{array}$} & \multicolumn{2}{|c|}{ HPV Infection } & \multirow{2}{*}{$\begin{array}{c}\mathrm{p}- \\
\text { value }\end{array}$} & \multicolumn{2}{|c|}{ HSV-2 Infection } & \multirow{2}{*}{$\begin{array}{c}\mathrm{p}- \\
\text { value }\end{array}$} \\
\hline & & & & $\begin{array}{l}\mathrm{HIV+}+ \\
\mathrm{n}=34\end{array}$ & $\begin{array}{l}\text { HIV- } \\
n=14\end{array}$ & & $\begin{array}{l}\mathrm{HIV+} \\
\mathrm{n}=43\end{array}$ & $\begin{array}{l}\text { HIV- } \\
\mathrm{n}=28\end{array}$ & & $\begin{array}{l}\mathrm{HIV+}+ \\
\mathrm{n}=44\end{array}$ & $\begin{array}{l}\text { HIV- } \\
\mathrm{n}=28\end{array}$ & & $\begin{array}{l}\mathrm{HIV+}+ \\
\mathrm{n}=79\end{array}$ & $\begin{array}{l}\text { HIV- } \\
n=48\end{array}$ & \\
\hline \multirow[t]{2}{*}{ MSP } & No & $43(41.0)$ & $33(31.4)$ & $15(34.9)$ & $8(24.2)$ & 0.143 & $21(48.8)$ & $12(36.4)$ & 0.352 & $19(44.2)$ & 7 (21.2) & 0.051 & $32(74.4)$ & $14(42.4)$ & 0.009 \\
\hline & Yes & $62(59.0)$ & $72(68.6)$ & $19(30.6)$ & $6(8.3)$ & 0.002 & $22(35.5)$ & $16(22.2)$ & 0.124 & $25(40.3)$ & $21(29.2)$ & 0.204 & $47(75.8)$ & $34(47.2)$ & 0.001 \\
\hline \multirow[t]{2}{*}{ HUTI } & No & $74(70.5)$ & $84(80.0)$ & $19(25.7)$ & $10(11.9)$ & 0.038 & $26(35.1)$ & $21(25.0)$ & 0.222 & $27(36.5)$ & $22(26.2)$ & 0.173 & $54(73.0)$ & $36(42.9)$ & 0.000 \\
\hline & Yes & $31(29.5)$ & $21(20.0)$ & $15(48.4)$ & $4(19.0)$ & 0.042 & $17(54.8)$ & $7(33.3)$ & 0.162 & $17(54.8)$ & $6(28.6)$ & 0.089 & $25(80.6)$ & $12(57.1)$ & 0.117 \\
\hline \multirow[t]{2}{*}{ Ulcers } & No & $93(88.6)$ & $98(93.3)$ & $30(32.3)$ & $12(12.2)$ & 0.001 & $35(37.6)$ & $25(25.5)$ & 0.087 & $39(41.9)$ & $24(24.5)$ & 0.014 & 68 (73.1) & $44(44.9)$ & 0.000 \\
\hline & Yes & $12(11.4)$ & $7 \quad(6.7)$ & $4(33.3)$ & $2(28.6)$ & 1.000 & $8(66.7)$ & $3(42.9)$ & 0.377 & $5(41.7)$ & $4(57.1)$ & 0.650 & 11 (91.7) & $4 \quad(57.1)$ & 0.117 \\
\hline \multirow[t]{2}{*}{ Vulval itching } & No & 81 (77.1) & $80(76.2)$ & $26(32.1)$ & $9(34.6)$ & 0.002 & $31(38.3)$ & $25(31.3)$ & 0.409 & $31(38.3)$ & $20(25.0)$ & 0.090 & $61(75.3)$ & $40(50.0)$ & 0.001 \\
\hline & Yes & $24(22.9)$ & $25(23.8)$ & $8(33.3)$ & $5(20.0)$ & 0.345 & $12(54.5)$ & $3(12.0)$ & 0.005 & $13(54.2)$ & $8(32.0)$ & 0.244 & $18(75.0)$ & $\begin{array}{ll}8 & (32.0)\end{array}$ & 0.004 \\
\hline \multirow[t]{2}{*}{ Vaginal discharge } & No & $59(56.2)$ & $61(58.1)$ & $19(32.2)$ & $7(11.5)$ & 0.008 & $22(37.3)$ & $15(24.6)$ & 0.167 & $25(42.4)$ & $18(29.5)$ & 0.183 & $43(72.9)$ & $34(55.7)$ & 0.059 \\
\hline & Yes & $46(43.8)$ & $44(41.9)$ & $15(32.6)$ & 7 (15.9) & 0.087 & $21(45.7)$ & $13(29.5)$ & 0.133 & $19(41.3)$ & $10(22.7)$ & 0.073 & $36(78.3)$ & $14(31.8)$ & 0.000 \\
\hline \multirow[t]{2}{*}{ Vaginal bleeding } & No & $100(95.2)$ & $99(94.3)$ & $32(32.0)$ & $10(10.1)$ & 0.000 & $40(40.0)$ & $27(27.3)$ & 0.072 & $42(42.0)$ & $28(28.3)$ & 0.054 & $74(74.0)$ & $46 \quad(46.5)$ & 0.000 \\
\hline & Yes & $5 \quad(4.8)$ & $6 \quad(5.7)$ & $2(40.0)$ & $4(66.7)$ & 0.567 & $3(60.0)$ & $1(16.7)$ & 0.242 & $2(40.0)$ & $0(0.0)$ & 0.182 & $5(100)$ & $2 \quad(33.3)$ & 0.061 \\
\hline \multirow[t]{2}{*}{ Pelvic Pain } & No & $97(92.4)$ & $80(76.2)$ & $33(34.0)$ & $11(13.8)$ & 0.003 & $40(41.2)$ & $21(26.3)$ & 0.040 & $39(40.2)$ & $20(25.0)$ & 0.038 & $72(74.2)$ & $39(48.8)$ & 0.001 \\
\hline & Yes & $\begin{array}{ll}8 & (7.6)\end{array}$ & $25(23.8)$ & $1(12.5)$ & $3(12.0)$ & 1.000 & $3(37.5)$ & $7(28.0)$ & 0.673 & $5(62.5)$ & $8(32.0)$ & 0.213 & $7(87.5)$ & $\begin{array}{ll}9 & (36.0)\end{array}$ & 0.017 \\
\hline \multirow[t]{2}{*}{ UCS } & No & $101(96.2)$ & $96(91.4)$ & $33(32.7)$ & $11(11.5)$ & 0.001 & $39(38.6)$ & $27(28.1)$ & 0.099 & $42(41.6)$ & $26(27.1)$ & 0.037 & $75(74.3)$ & $44(14.6)$ & 0.000 \\
\hline & Yes & $4 \quad(3.8)$ & $9(8.6)$ & $1(20.0)$ & $3(33.3)$ & 1.000 & $2(50.0)$ & 1 (11.1) & 0.203 & $2(50.0)$ & $2(22.2)$ & 0.530 & $4(100)$ & $4 \quad(44.4)$ & 0.105 \\
\hline \multirow[t]{5}{*}{ Pap smear result } & None & $36(34.3)$ & $60(57.1)$ & -- & -- & - & $11(30.6)$ & $12(20.0)$ & 0.324 & $8(22.2)$ & $18(30.0)$ & 0.482 & $22(61.1)$ & $23(38.3)$ & 0.036 \\
\hline & Cervicitis & $15(14.3)$ & $19(18.1)$ & -- & -- & -- & $4(26.7)$ & $6(31.6)$ & 1.000 & $7(46.7)$ & $2(10.5)$ & 0.022 & $12(80.0)$ & $9 \quad(47.4)$ & 0.079 \\
\hline & ASCUS & $20(19.9)$ & $12(11.4)$ & -. & -- & - & $8(40.0)$ & $3(25.0)$ & 0.465 & $8(40.0)$ & $4(33.3)$ & 1.000 & $19(95.0)$ & $7 \quad(58.3)$ & 0.019 \\
\hline & LSIL & $20(19.9)$ & $8 \quad(7.6)$ & -- & - & - & $8(40.0)$ & $5(62.5)$ & 0.410 & $11(55.0)$ & $3(37.5)$ & 0.678 & $14(70.0)$ & $\begin{array}{ll}5 & (62.5)\end{array}$ & 1.000 \\
\hline & HSIL & $14(13.3)$ & $\begin{array}{ll}6 & (5.7)\end{array}$ & -- & -- & - & $12(85.7)$ & $2(33.3)$ & 0.037 & $10(71.4)$ & 1 (16.7) & 0.050 & $12(85.7)$ & $\begin{array}{ll}4 & (66.7)\end{array}$ & 0.549 \\
\hline \multirow[t]{8}{*}{ Infection } & None & $12(11.4)$ & $43(41.0)$ & $4(3.8)$ & $5(4.7)$ & 0.092 & -- & -- & -- & -- & -- & -- & -- & -- & -- \\
\hline & EBV & $2(1.9)$ & $3(2.9)$ & $0(0.0)$ & $0(0.0)$ & 1.000 & -- & -- & -- & - & -- & -- & -- & -- & -- \\
\hline & HPV & $11(10.5)$ & $10(9.5)$ & $4(3.8)$ & $0(0.0)$ & 0.090 & -- & -- & -- & - & - & -- & -- & -- & -- \\
\hline & HSV-2 & $33(31.4)$ & $16(15.2)$ & $3(2.9)$ & $2(1.9)$ & 1.000 & -- & -- & -- & - & - & -- & -- & -- & -- \\
\hline & EBV+HSV-2 & $14(13.3)$ & $15(14.3)$ & $6(5.7)$ & $3(2.9)$ & 0.245 & -- & -- & -- & - & -- & -- & -- & -- & -- \\
\hline & $H P V+H S V-2$ & $6(5.7)$ & $8(7.6)$ & $3(2.9)$ & $0(0.0)$ & 0.054 & -- & -- & -- & -- & -. & -- & -- & -- & -- \\
\hline & EBV+HPV & $1(1.0)$ & $1(1.1)$ & $0(0.0)$ & $0(0.0)$ & 1.000 & -- & -- & -- & -- & -- & -- & -- & -- & -- \\
\hline & $\mathrm{EBV} / \mathrm{HPV} / \mathrm{HSV}-2$ & $26(24.8)$ & $9(8.6)$ & $14(13.3)$ & $4(3.8)$ & 0.711 & -- & -- & -- & -- & -- & -- & - & -- & -- \\
\hline
\end{tabular}

Keys: N.F.E; No formal education, M.S.P; Multiple sexual partners, Sec; Secondary; D/S/W; Divorced/Separated/Widow, Abn; Abnormal, Ppt; participants, HUTI; History of urogenital infection; UCS; Uptake of cervical screening.

Prevalence of SIL in acute and chronic viral infection The ratio of chronic HPV infection and acute HPV infection in HIV+ and HIV- women were 2.7:1 and 7:1, respectively. In $\mathrm{HIV}+$ women with SIL, higher prevalence of chronic HSV-2 and EBV infections $(70.6 \%$ and $55.9 \%$ ) were observed than acute infections $(50 \%$ and $17.6 \%$, respectively) while lower prevalence of chronic
HPV infection $(23.5 \%)$ was observed than acute HPV infection (41.2\%). In HIV- women with SIL, higher prevalence of chronic HPV, HSV-2 and EBV infections (21.4, 64.3 and 50\%) were observed than acute infections (14.3, 35.7 and $21.4 \%$, respectively). The number of women who were positive for both IgG and IgM antibodies were higher in HIV+ participants when compare with HIV- women (table 2).

Table 2: Viral antibody positivity among HIV- and HIV+ participants in relation to some classes of Pap smear

\begin{tabular}{|c|c|c|c|c|c|c|c|c|c|c|}
\hline \multirow[t]{2}{*}{ Groups } & \multirow[t]{2}{*}{$\mathrm{N}=\mathbf{2 1 0}$} & \multicolumn{2}{|c|}{ HPV } & \multicolumn{2}{|c|}{ HSV-2 } & \multicolumn{2}{|c|}{ EBV } & \multirow{2}{*}{$\begin{array}{c}\text { HPV } \\
\text { IgG+IgM }\end{array}$} & \multirow{2}{*}{$\begin{array}{c}\text { HSV-2 } \\
\text { IgG+IgM }\end{array}$} & \multirow{2}{*}{$\begin{array}{c}\text { EBV } \\
\text { IgG+IgM }\end{array}$} \\
\hline & & IgG & IgM & IgG & IgM & IgG & IgM & & & \\
\hline Cut-off valu & & 1.071 & 0.438 & 1.520 & 0.102 & 1.570 & 1.030 & & & \\
\hline \multicolumn{11}{|c|}{ HIV Seropositive } \\
\hline HSIL & $14(13.3)$ & $4(28.6)$ & $6(42.9)$ & $11(78.6)$ & $5(35.7)$ & $11(78.6)$ & $3(11.5)$ & $0(0.0)$ & $4 \quad(28.6)$ & $2(11.5)$ \\
\hline LSIL & $20(19.0)$ & $4(20.0)$ & $8(40.0)$ & $13(65.0)$ & $12(60.0)$ & $8(40.0)$ & $3(18.8)$ & $1(5.0)$ & $12(60.0)$ & $3(12.5)$ \\
\hline ASCUS & $20(19.0)$ & $7(35.0)$ & $4(20.0)$ & $17(85.0)$ & $16(80.0)$ & $8(40.0)$ & $2(10.0)$ & $3(15.0)$ & $14(70.0)$ & $2(10.0)$ \\
\hline Cervicitis & $15(14.3)$ & $2(13.3)$ & $7(46.7)$ & $5(33.3)$ & $7(46.7)$ & $1(6.7)$ & $3(20.0)$ & $2(13.3)$ & $0(0.0)$ & $0(0.0)$ \\
\hline NILM & $36(34.3)$ & $1(2.8)$ & $6(16.7)$ & $9(25.0)$ & $13(36.1)$ & $8(40.0)$ & $3(4.8)$ & $0(0.0)$ & $0(0.0)$ & $0(0.0)$ \\
\hline \multicolumn{11}{|c|}{ HIV Seronegative } \\
\hline HSIL & $6(5.7)$ & $1(16.7)$ & $0(0.0)$ & $4(66.7)$ & $2(33.3)$ & $2(33.3)$ & $1(16.7)$ & $0(0.0)$ & $2(33.3)$ & $1(16.7)$ \\
\hline LSIL & $8(7.6)$ & $2(25.0)$ & $2(25.0)$ & $5(62.5)$ & $3(37.5)$ & $5(62.5)$ & $2(25.0)$ & $1(12.5)$ & $3(37.5)$ & $2(25.0)$ \\
\hline ASCUS & $12(11.4)$ & $3(25.0)$ & $1(8.3)$ & $5 \quad(41.7)$ & $3(60.0)$ & $3(33.3)$ & $1(8.3)$. & $0(0.0)$ & $1(8.3)$ & $1(8.3)$ \\
\hline Cervicitis & $19(18.1)$ & $1(5.26)$ & $1(5.3)$ & $4 \quad(21.1)$ & $6(31.6)$ & $5(26.3)$ & $1(5.3)$ & $0(0.0)$ & $1(5.3)$ & $0(0.0)$ \\
\hline NILM & $60(57.1)$ & $16(26.7)$ & $2(3.3)$ & $15(25.0)$ & $12(20.0)$ & $12(31.7)$ & $2(3.3)$ & $0(0.0)$ & $4 \quad(6.7)$ & $1(1.7)$ \\
\hline $\mathrm{HIV}+(n=105$ & & $18(17.1)$ & $31(29.5)$ & $55(52.4)$ & $53(50.5)$ & $36(34.3)$ & $14(10.3)$ & $3(2.9)$ & $30(28.6)$ & $7(6.2)$ \\
\hline HIV- $(n=105$ & & $23(15.2)$ & $6(5.7)$ & $33(31.4)$ & $26(24.8)$ & $27(25.7)$ & $7(6.7)$ & $1(1.0)$ & $11(10.5)$ & $5(4.8)$ \\
\hline $\mathrm{HIV}+$ vs HI & - p-value & 0.4865 & $<0.0001$ & 0.0032 & 0.0002 & 0.2282 & 0.1664 & 0.6214 & 0.0015 & 0.7677 \\
\hline
\end{tabular}




\section{Discussion}

The prevalence of cervical lesions in HIV+ and HIVwomen in this study is similar to the $56 \%$ and $12.6 \%$ reported among HIV+ and HIV- women in North Central Nigeria by Lawal et al., respectively ${ }^{23}$ but lower than the $61.1 \%$ and $76 \%$ reported in HIV+ women at Tanzania and Zambia, respectively ${ }^{11,24}$. In this study, te prevalence of SIL is higher than the prevalence reported in Etiopian HIV+ women (13.6\%) and HIV- women $(5.2 \%)$, respectively ${ }^{25}$. Studies have shown that HIV infection increases the risk for CIN (SIL) by 2.3 to 5 times ${ }^{26-28}$. This is similar to the findings of this study which showed that the prevalence of SIL was 2.4 times higher in HIV+ women than in HIV- women. The difference in the prevalence of SIL between HIV+ and HIV-participants could be accrued to other viral infections apart from HPV infection. Such viruses may include EBV and HSV-2. The prevalence of HSV-2 infection in this study is similar to the prevalence of $85 \%$ and $53 \%$ observed in HIV+ and HIV- participants in Uganda $^{29}$. Similar to the findings of Nakku-Jobba et al., age, level of education, number of sexual partners and presence of ulcers were major correlates of HSV-2 infections ${ }^{29}$. A recent study revealed that HSV-2 seropositive women have Ca risk of 2- to 9-fold ${ }^{30}$. The increasing prevalence of the virus with disease state suggests that HSV-2 plays a role in cervical carcinogenesis. Additionally, the co-existence of HSV-2 and HPV is believed to be associated with higher risk for $\mathrm{Ca}$. This is evident in a study which shows that the relative risk (RR) for Ca among HSV-2, HPV and HSV-2/HPV positive groups are $2.79,2.98$ and $3.44(95 \% \mathrm{Cl}: 1.31-5.96,1.23-7.20$ and 1.50-7.86), respectively ${ }^{31}$.

Interestingly, the prevalence of EBV infection was associated with older age in HIV+ women while the prevalence of the virus was associated with younger age in HIV- women. The divergence in age related prevalence of the virus may account for the difference in the prevalence of SIL between the two groups. This is underscored by the fact that the prevalence of SIL in HIV+ women was associated with older age as well. The relationship between older age and prevalence of EBV has early been reported ${ }^{20}$. Unlike in HIV- women, increasing prevalence of EBV infection was associated with disease state in HIV+ women, with the highest prevalence in HSIL. Similar reports of have been made in earlier studies ${ }^{20-21}$. However, these studies were not explicit on the HIV status of their entire participants.
Though the prevalence of HPV infection was associated with younger age both in HIV+ and HIV- women, its prevalence was associated with higher disease state in HIV+ women. This again demonstrates the importance of EBV and HPV persistence in cervical carcinogenesis, especially in immunocompromised individuals. Studies have shown that the absence or reduced antiviral T-cell response in HIV infection favours superinfections, co-infections, persistence, replication and reactivation of latent oncoviruses ${ }^{32,33}$. This might explains why HIV+ women had higher EBV/HPV associated SIL than HIV- women. Studies have shown that the presence of EBV latent membrane protein 1 and HPV is associated with aggressive and poorly differentiated squamous cell carcinomas phenotype $\mathrm{e}^{20,34,35}$. This may be associated with the higher prevalence of SIL observed in HIV+ women. The high prevalence of SIL in HIV+ women with tri-infection could be due to immune exhaustion, since these women had a lower expression of Ki67 in HSIL than their HIV- counterparts ${ }^{18}$. Although the prevalence of HSV-2 is lower in HIV- women than their HIV+ counterparts, such HIV- women are at risk of acquiring HIV infections, especially those with acute HSV-2 infections ${ }^{36}$.

\section{Conclusion}

This study revealed that the prevalence of viral mono-infections and co-infections, and SIL were higher in HIV+ women than in HIV- women. It also revealed that higher prevalence of SIL in viral tri-infection than in bi-infection and mono-infection both in HIV + and HIV- women. It suggests that the high prevalence of SIL in HIV+ women could be associated with viral co-infections.

\section{Acknowledgements}

Special thanks are to staff of HIV Counseling and Testing and Family planning clinics State Hospital Abeokuta for their technical assistance.

\section{Conflict of interest}

None declared

\section{References}

1. Arbyn M, Castellsague X, de Sanjose S, Bruni L, Saraiya M, Bray F, et al. Worldwide burden of cervical cancer in 2008. Annals of Oncology 2011; 22 (12): 2675 2686.

2. Shi Y, Peng SL, Yang LF, Chen X, Tao YG, Cao 
.Co-infection of Epstein-Barr virus and human papillomavirus in human tumorigenesis. Chinese Journal of Cancer. 2016; 35:16

3. Ogembo RK, Gona PN, Seymour AJ, Park HSM, Bain PA, MarandaL, et al. Prevalence of Human Papillomavirus Genotypes among African Women with Normal Cervical Cytology and Neoplasia: A Systematic Review and Meta-Analysis. PLoS One 2015; 10(4): e0122488.

4. Agaba PA, Thacher TD, Ekwempu CC, Idoko JA. Cervical dysplasia in Nigerian women infected with HIV. International Journal of Gynaecology and Obstetrics 2009; 107 (2): 99-102

5. Omar T, Schwartz S, Hanrahan C, Modisenyane T, Tshabangu N, Golub JE, et al. Progression and regression of premaligant cervical lesions in HIV-infected women from Soweto: a prospective cohort. AIDS 2010; 25 (1): 87-94.

6. Chaturvedi AK, Madeleine MM, Biggar RJ, Engels EA. Risk of human papillomavirus -associated cancers among persons with AIDS. Journal National Cancer Institute 2009; 101(16): 1120-1130.

7. Moscicki AB1, Ellenberg JH, Farhat S, Xu $\mathrm{J}$. Persistence of human papillomavirus infection in HIV-infected and uninfected adolescent girls: risk factors and differenced, by phylogenetic type. Journal of Infectious Diseases 2004; 190 (1) 37-45.

8. Cirac A, Stützle S, Dieckmeyer M, Adhikary D, Moosmann A, Körber N. Epstein-Barr virus strain heterogeneity impairs human T-cell immunity. Cancer Immunology and Immunotherapy. 2018a; 67 (4): 663-674.

9. Chirenje ZM. HIV and cancer of the cervix. Best Practice \& Research: Clinical Obstetrics \& Gynaecology. 2005; 19 (2): 269-276.

10. Denslow SA, Rositch AF, Firnhaber C, Ting J, Smith JS. Incidence and progression of cervical lesions in women with HIV: A systematic global review. International Journal of STD \& AIDS 2014; 25 (3): 163-177.

11. Stier EA. Human papillomavirus-related diseases in HIV-infected individuals. Current Opinion in Oncology 2009; 20 (5): 541-546.

12. Parham GP, Sahasrabuddhe VV, Mwanahamuntu MH, Shepherd BE, Hicks ML, Stringer EM, et al. Prevalence and predictors of squamous intraepithelial lesions of the cervix in HIV-infected women in Lusaka, Zambia. Gynecologic Oncology 2006; 103 (3): 1017-1022.

13. Smith JS, Herrero R, Bosetti C, Munoz N, Bosch FX, Eluf-Neto J, Castellsague X, Meijer CJ, Van den Brule AJ, Franceschi $S$ et al: Herpes simplex virus-2 as a human papillomavirus cofactor in the etiology of invasive cervical cancer. Journal of National Cancer Institute 2002, 94(21):1604-1613.

14. Daling JR, Madeleine MM, McKnight B, Carter JJ, Wipf GC, Ashley R, et al. The relationship of human papillomavirus-related cervical tumors to cigarette smoking, oral contraceptive use, and prior herpes simplex virus type 2 infection. Cancer Epidemiol Prev Biomarkers. 1996; 5:541-548.

15. Bashyal R, Dali S. Study on koilocytosis, $\mathrm{X}$-chromatin and HSV-2 in cervical smears in Nepal. Nepal Medical College Journal: NMCJ. 2004; 6:45-48 PubMed .

16. Silver MI, Paul P, Sowjanya P, Ramakrishna G, Vedantham H, Kalpana B, et al. Shedding of Epstein Barr virus and cytomegalovirus from the genital tract of women in a periurban community in Andhra Pradesh. India. J Clin Microbiol. 2011; 49(7): 2435 PubMed -2439. 17. Santos NB, Villanova FE, Andrade PM, Ribalta J, Focchi J, Otsuka AY, et al. Epstein-Barr virus detection in invasive and pre-invasive lesions of the uterine cervix. Oncology Reports. 2009; 21(2): 403 PubMed -405. 18. Abudoukadeer, M. Niyazi, A. Aikula, M. Kamilijian, X. Sulaiman, A. Mutailipu, et al. Association of EBV and HPV co-infection with the development of cervical cancer in ethnic Uyghur women. European Journal Gynaecological and Oncology. 2015; 36 (5): 546-550. 19. Aromseree S, Pientong C, Swangphon P, Chaiwongkot A, Patarapadungkit N, Kleebkaow P, et al. Possible contributing role of Epstein-Barr virus (EBV) as a cofactor in human papillomavirus (HPV) associated cervical carcinogenesis. Journal of Clinical Virology. 2015;73:70-6.

20. Khenchouche A, Sadouki N, Boudriche A, Houali K, Graba A, Ooka T, et al. Human papillomavirus and Epstein-Barr virus co-infection in cervical carcinoma in Algerian women. Virology Journal. 2013;10:340. 21. de Lima MA, Neto PJ, Lima LP, Júnior JG, Junior AG, Teodoro IP, et al. Association between Epstein-Barr virus (EBV) and cervical carcinoma: a meta-analysis. Gynecol Oncol 2018; 148: 317-28 PubMed .

22. Bashorun A, Nguku P, Kawu I, Ngige E, Ogundiran A, Sabitu K, et al. A description of HIV prevalence trends in Nigeria from 2001 to 2010: what is the progress, where is the problem? The Pan African Medical Journal 2014; 18 (Suppl 1): 3. doi: 10.11694/pamj. supp.2014.18.1.4608 PubMed

23. Lawal I, Agida TE, Offiong RA, Oluwole PO. Cervical cytology among HIV positive and HIV nega- 
tive women in a tertiary Hospital in North Central Nigeria. A comparative Study. Annals of Medical and Health Science Research. 2017; 7 (5): 308-311.

24. Kafuruki L, Rambau PF, Massinde A, Masalu N. Prevalence and predictors of Cervical Intraepithelial Neoplasia among HIV infected women at Bugando Medical Centre, Mwanza-Tanzania. Infectious Agents and Cancer, 2013; 8: 45S. http://doi.org/10.1186/17509378-8-45

25. Getinet M, Gelaw B, Sisay A, Mahmoud EA, Assefa A. Prevalence and predictors of Pap smear cervical epithelial cell abnormality among HIV-positive and negative women attending gynecological examination in cervical cancer screening center at Debre Markos referral hospital, East Gojjam, Northwest Ethiopia. Clinical Pathology (2015) 15:16.

26. Duerr A, Kieke B, Warren D, Shah K, Burk R, Peipert JF, et al. Human papillomavirus-associated cervical cytologic abnormalities among women with or at risk of infection with human immunodeficiency virus. American Journal of Obstetrics \& Gynecology. 2000; 184 (4): 584 PubMed -590.

27. Grulich AE1, van Leeuwen MT, Falster MO, Vajdic CM. Incidence of cancers in people with HIV/ AIDS compared with immunosuppressed transplant recipients: a meta-analysis. Lancet. 2007; 370 (9581): 59-67.

28. Chambuso RS, Shadrack S, Lidenge SJ, Mwakibete N, Medeiros RM. Influence of HIV/AIDS on Cervical Cancer: A Retrospective Study From Tanzania. Journal of Global Oncology 2017; 3 (1): 72-78.

29. Nakku-Joloba E, Kambugu F, Wasubire J, Kimeze J, Salata R, Albert JM, et al. Sero-prevalence of herpes simplex type 2 virus (HSV-2) and HIV infection in Kampala, Uganda. African Health Sciences 2014; 14 (4): 782-789.

30. Guidry JT, Scott RS. The interaction between human papillomavirus and other viruses. Virus Research. 2017; 231: 139-147 PubMed .

31. Li S, Wen X. Seropositivity to herpes simplex virus type 2 , but not type 1 is associated with cervical cancer: NHANES (1999-2014). BMC cancer 2017; 17 (1): 726 PubMed .

32. Fu S, Shi N, Wheler J, Naing A, Janku F, Piha-Paul S. Characteristics and outcomes for patients with advanced vaginal or vulvar cancer referred to a phase I clinical trials program. Gynecologic Oncology Research and Practice. 2015; 2: 10. doi:10.1186/s40661-0150018-x

33. Yang F, Liu Q, Hu CM. Epstein-Barr virus-encoded LMP1 increases miR-155 expression, which promotes radioresistance of nasopharyngeal carcinoma via suppressing UBQLN1. European Review for Medical and Pharmacological Sciences. 2015; 19 (23): 4507-4515.

34. Al-Thawadi H, Ghabreau L, Aboulkassim T, Yasmeen A, Vranic S, Batist G, Al Moustafa AE. co-incidence of epstein-Barr Virus and high-risk human Papillomaviruses in cervical cancer of syrian Women. Frontiers in Oncology. 2018;8:250.

35. Al Moustafa A-E, Ahmed HG, Wulf G and Sultan AA. Editorial: EBV-Associated Carcinomas: Presence, Role, and Prevention Strategies. Frontiers in Oncology. 2018; 8: 528.

36. Looker KJ, Elmes JAR, Gottlieb SL, Schiffer JT, Vickerman P, Turner KME. Effect of HSV-2 infection on subsequent HIV acquisition: an updated systematic review and meta-analysis. Lancet Infectious Disease 2017; 17 (12): 1303-1316 